Proyecciones

Vol. $12 \mathrm{~N}^{\circ} 2, \mathrm{pp} .161-169$ December 1993

Universidad Católica del Norte

Antofagasta - Chile

\title{
LOCAL STABILITY RESULTS ON A MODEL FOR TYPHOID FEVER WITH A CORE GROUP*
}

\author{
JORGE GONZALEZ-GUZMAN \\ BETSABE GONZALEZ-YAÑEZ \\ Universidad Católica de Valparaíso, Valparaíso, Chile.
}

\begin{abstract}
A SIRS epidemiological model with two subpopulation and vital dynamics is analized. Both subpopulations are considered constant by assuming that the birth and the death rate are equal. We analize the case where one subpopulation is a core, that is, a very infectious small group, responsible for a big fraction of the incidence. For this case a threshold is determined and the corresponding equilibrium points for the four dimensional system are shown to be locally stable by means of the classical Liapunov theorem. This system models the dynamics of typhoid fever, where the core is the group of food manipulators. Computer simulation are used to estimate the effect of vaccination on the population.
\end{abstract}

* Research supported by FONDECYT under Project 0028-92 and by DGIP under Project $124.707 / 92$. 


\section{Introduction.}

The spread of an infectious disease in a population depends not only on the character of the disease, but also on the structure and behavior of the population. Therefore the study of heterogeneous epidemic models is very important. Many previous results have been obtained in this direction. Hethcote [3] analyzed the behavior of solutions of a SIR model with $\mathrm{n}$ subpopulations, but without vital dynamics (birtlıs and deaths). E.Beretta and V.Capasso [8] give conditions for global stability in a SIR model with n subpopulations. For a SEIRS model with n subpopulations, Thieme [4] showed global asymptotic stability under certain conditions. Hetlicote and Thieme [5] analyze two different SIRS models with n subpopulations and show local stability for the endemic equilibrium. The global stability of the endemic equilibrium for the two models is still an open problem.

In the present paper we identify a threshold quantity for a SIRS model with two subpopulations and show the local stability for both the disease free and the endemic equilibrium. We consider the case of a core-noncore model [1], where the infectious contacts of the core people with the noncore ones are responsible for most of the total incidence. Our aim is to model the spread of typhoid fever in a modern big city, where many people eat in restaurants. In this case the food manipulators constitute the core group.

\section{Tlie Model.}

Let $\mathrm{N}$ be the total size of the population, $N_{1}$ the size of the core and $N_{2}$ the size of the noncore. Then $N=N_{1}+N_{2} ; N_{i}=S_{i}+I_{i}+R_{i}, i=1,2$, where S, I, R denote the number of susceptibles, infectious and removed of each subpopulation. We assume that both subpopulations $N_{1}$ and $N_{2}$ are constant by setting the birth rate equal to the death rate. Note that, in the case of the core group, the birth and the death rate have the meaning of a rate of change of the food manipulators, not necesarly equal to the birth and deatl rate of the population. Let $x_{i}=I_{i} / N_{i}$ , $y_{i}=R_{i} / N_{i}, i=1,2 ;\left(\lambda_{i j}\right)$ is the transmission matrix, that is, $\lambda_{i j}$ is the average number of infectious contacts (also called adequate contacts, see [1]) of an infective in group $j$ with persons in group $i$ per unit time. Let $p=N_{1} / N_{2}, \delta_{i}$ the birtl (or death ) rate of subpopulation $i, \gamma$ is the recovery removal rate $(1 / \gamma$ is the average infectious period ), $w_{i}$ is the rate of loss of temporary immunity for group $i$ and $v_{i}$ is the immunization rate by vaccination in group $i$. We can accept different values of duration of immunity because the vaccination strategy on both groups can be different (for example, food manipulators call get double dosis vaccine).

The model is then given by the following four dimensional system of autonomous differential equations : 


$$
\begin{aligned}
& x_{1}^{\prime}=\left(1-x_{1}-y_{1}\right)\left(\lambda_{11} x_{1}+\frac{\lambda_{12}}{p} x_{2}\right)-x_{1}\left(\delta_{1}+\gamma\right) \\
& y_{1}^{\prime}=v_{1}+\left(\gamma-v_{1}\right) x_{1}-\left(v_{1}+\delta_{1}+w_{1}\right) y_{1} \\
& x_{2}^{\prime}=\left(1-x_{2}-y_{2}\right)\left(\lambda_{22} x_{2}+\lambda_{21} p x_{1}\right)-x_{2}\left(\delta_{2}+\gamma\right) \\
& y_{2}^{\prime}=v_{2}+\left(\gamma-v_{2}\right) x_{2}-\left(v_{2}+\delta_{2}+w_{2}\right) y_{2}
\end{aligned}
$$

with the conditions: $x_{i}, y_{i} \geq 0 ; x_{i}+y_{i} \leq 1, i=1,2$.

Let $D$ be the domain $x_{i}, y_{i} \geq 0 ; x_{i}+y_{i} \leq 1, i=1,2$ in the four dimensional space $R^{4}$. It is easy to see, by applying Nagunos's Lemma, that domain D is positively invariant for system (1). This system satisfies the existence, uniquenes and prolongability conditions so that solutions of (1) starting in $D$ exists for all time and remain in $D$. Thus the nodel is well-possed.

The qualitative analyse of system (1) is very difficult because of the dimension, even the equilibrium points can not be found explicity. But, if we assume that $\lambda_{12}=\lambda_{22}=0$, then system (1) become mathematically tractable. This supposition means that the model consider only the effect of the core on the spread of the disease in the population.

By analyzing system (1) we obtain the following threshold parameter :

$$
\sigma=\frac{\lambda_{11}\left(\delta_{1}+w_{1}\right)}{\left(\delta_{1}+\gamma\right)\left(v_{1}+\delta_{1}+w_{1}\right)}
$$

\section{Theorem 1.}

a) If $\sigma \leq 1$, then the only equilibrium point of system (1) is the disease-free equilibrium :

$$
E_{1}=\left(0, v_{1} /\left(v_{1}+\delta_{1}+w_{1}\right), 0, v_{2} /\left(v_{2}+\delta_{2}+w_{2}\right)\right)
$$

which is locally stable.

b) If $\sigma>1$, then there are two equilibrium points in $D$ : the disease free equilibrium $E_{1}$ and the endemic equilibrium point $E_{2}=\left(x_{1}^{*}, y_{1}^{*}, x_{2}^{*}, y_{2}^{*}\right)$, 
where :

(4)

$$
\begin{aligned}
& x_{1}^{*}=\frac{\lambda_{11}\left(\delta_{1}+w_{1}\right)-\left(\delta_{1}+\gamma\right)\left(v_{1}+\delta_{1}+w_{1}\right)}{\lambda_{11}\left(\delta_{1}+w_{1}+\gamma\right)} \\
& y_{1}^{*}=\frac{\lambda_{11} \gamma+\left(\delta_{1}+\gamma\right)\left(v_{1}-\gamma\right)}{\lambda_{11}\left(\delta_{1}+w_{1}+\gamma\right)} \\
& x_{2}^{*}=\frac{p x_{1}^{*} \lambda_{21}\left(w_{2}+\delta_{2}\right)}{\left(\delta_{2}+\gamma\right)\left(v_{2}+w_{2}+\delta_{2}\right)+p x_{1}^{*} \lambda_{21}\left(w_{2}+\delta_{2}+\gamma\right)} \\
& y_{2}^{*}=\frac{v_{2}\left(\delta_{2}+\gamma\right)+p x_{1}^{*} \lambda_{21} \gamma}{\left(\delta_{2}+\gamma\right)\left(v_{2}+w_{2}+\delta_{2}\right)+p x_{1}^{*} \lambda_{21}\left(w_{2}+\delta_{2}+\gamma\right)}
\end{aligned}
$$

This endemic point is locally stable while the disease-free equilibrium point $E_{1}$ is unstable.

Proof. First we note that always $E_{1} \in D$ but $E_{2} \in D$ if and only if :

$$
\lambda_{11}\left(\delta_{1}+w_{1}\right)>\left(\delta_{1}+\gamma\right)\left(v_{1}+\gamma\right)\left(v_{1}+\delta_{1}+w_{1}\right)
$$

This last condition is equivalent to the condition $\sigma>1$. In order to analize the local stability of the equilibrium points we calculate the Jacobian Matrix of system (1):

$$
\left(\begin{array}{cccc}
\lambda_{11}\left(1-2 x_{1}-y_{1}\right)-\delta_{1}-\gamma & -\lambda_{11} x_{1} & 0 & 0 \\
\gamma-v_{1} & -\left(v_{1}+w_{1}+\delta_{1}\right) & 0 & 0 \\
\lambda_{21} p\left(1-x_{2}-y_{2}\right) & 0 & -\lambda_{21} p x_{1}-\delta_{1}-\gamma & -\lambda_{21} p x_{1} \\
0 & 0 & \gamma-v_{2} & -\left(v_{2}+w_{2}+\delta_{2}\right)
\end{array}\right)
$$

Evaluating in equilibrium point $E_{1}$ :

$$
\left(\begin{array}{cccc}
\frac{\lambda_{11}\left(1-v_{1}\right.}{\left(v_{1}+w_{1}+\delta_{1}\right)-\delta_{1}-\gamma} & 0 & 0 & 0 \\
\gamma-v_{1} & -\left(v_{1}+w_{1}+\delta_{1}\right) & 0 & 0 \\
\frac{\lambda_{21} p\left(1-v_{2}\right.}{\left.\left(v_{2}+w_{2}+\delta_{2}\right)\right)} & 0 & -\delta_{1}-\gamma & 0 \\
0 & 0 & \gamma-v_{2} & -\left(v_{2}+w_{2}+\delta_{2}\right)
\end{array}\right)
$$


The eigenvalues of this matrix are :

$$
\begin{aligned}
& z_{1}=-\left(v_{2}+w_{2}+\delta_{2}\right) \\
& z_{2}=-\left(\delta_{1}+\gamma\right) \\
& z_{3}=-\left(v_{1}+w_{1}+\delta_{1}\right) \\
& z_{4}=\frac{\lambda_{11}\left(w_{1}+\delta_{1}\right)}{v_{1}+w_{1}+\delta_{1}}-\delta_{1}-\gamma
\end{aligned}
$$

Note that $z_{1}, z_{2}$, and $z_{3}$ are always negative and $z_{4}$ is negative if and only if $\sigma<1$.

Since : $\lambda_{11}\left(1-2 x_{*}-y_{1}\right)-\delta_{1}-\gamma=-\lambda_{11} x_{*}$ the Jacobian matrix evaluated in point $E_{2}$ is :

$$
\left(\begin{array}{cccc}
-\lambda_{11} x_{1}^{*} & -\lambda_{11} x_{1}^{*} & 0 & 0 \\
\gamma-v_{1} & -\left(v_{1}+w_{1}+\delta_{1}\right) & 0 & 0 \\
\lambda_{21} p\left(1-x_{2}^{*}-y_{2}^{*}\right) & 0 & -\lambda_{21} p x_{1}^{*}-\delta_{1}-\gamma & -\lambda_{21} p x_{1}^{*} \\
0 & 0 & \gamma-v_{2} & -\left(v_{2}+w_{2}+\delta_{2}\right)
\end{array}\right)
$$

Hence, the characteristic polynomial decomposes in the two following quadratic equations :

$$
\begin{aligned}
& z^{2}+\left(v_{1}+w_{1}+\delta_{1}+\lambda_{11}\right) z+\lambda_{11} x_{1}^{*}\left(w_{1}+\delta_{1}+\gamma\right)=0 \\
& z^{2}+\left(\lambda_{21} p x_{1}+\delta_{1}+\delta_{2}+v_{2}+w_{2}+\gamma\right) z+ \\
& +\lambda_{21} p x_{1}^{*}\left(\delta_{2}+w_{2}+\gamma\right)+\left(\delta_{1}+\gamma\right)\left(v_{2}+w_{2}+\delta_{2}\right)=0
\end{aligned}
$$

Since the coefficients of this polynomials are positiv, the eigenvalues have negative real parts.

\section{Parameter Estination and Vaccination Strategies by Computer Simulation.}

Let us suppose that, without vaccination, the endemic values of both subpopulations are equal, and that in this case, $w_{1}=w_{2}$ and $\delta_{1}=\delta_{2}$. Then, 
using formulae (4) :

$$
x_{1}^{*}=\frac{p x_{1}^{*} \lambda_{21}\left(w_{2}+\delta_{2}\right)}{\left(\delta_{2}+\gamma\right)\left(v_{2}+w_{2}+\delta_{2}\right)+p x_{1}^{*} \lambda_{21}\left(w_{2}+\delta_{2}+\gamma\right)}
$$

thus :

$$
\begin{aligned}
& 1=\frac{p \lambda_{21}\left(w_{1}+\delta_{1}\right)}{\left(\delta_{1}+\gamma\right)\left(w_{1}+\delta_{1}\right)+p x_{1}^{*} \lambda_{21}\left(w_{1}+\delta_{1}+\gamma\right)} \\
& 1=\frac{p \lambda_{21}\left(w_{1}+\delta_{1}\right)}{\left(\delta_{1}+\gamma\right)\left(w_{1}+\delta_{1}\right)+p \frac{\lambda_{11}\left(\delta_{1}+u_{1}\right)-\left(\delta_{1}+\gamma\right)\left(v_{1}+\delta_{1}+w_{1}\right)}{\lambda_{11}\left(\delta_{1}+w_{1}+\gamma\right)} \lambda_{21}\left(w_{1}+\delta_{1}+\gamma\right)} \\
& 1=\frac{p \lambda_{21} \lambda_{11}}{\lambda_{11}\left(\delta_{1}+\gamma\right)+p \lambda_{21}\left(\lambda_{11}-\delta_{1}-\gamma\right)}
\end{aligned}
$$

thus:

$$
\lambda_{11}=p \lambda_{21}
$$

We use the estimation of the total prevalence given in [6]. Then:

$$
x_{1}^{*}=\frac{\left(\delta_{1}+w_{1}\right)\left(\lambda_{11}-\delta_{1}-\gamma\right)}{\lambda_{11}\left({ }_{1}+w_{1}+\gamma\right)}=0.007667
$$

hence:

$$
\lambda_{11}=\frac{\left(\delta_{1}+w_{1}\right)\left(\delta_{1}+\gamma\right)}{0.99233\left(\delta_{1}+w_{1}\right)-0.007667 \gamma}
$$

Now, considering that $[6]: \delta_{1}=0.02 ; \gamma=0.111$ and that the mean duration of the vaccine lias been estimated in 5 years, that is : $w_{1}=w_{2}=0.2$, and that we can estimate for Valparaiso : $p=0.017$, we obtain funally :

$$
\lambda_{11}=0.1325 ; \lambda_{21}=7.794
$$

Thus, the set of parameter values we use to model the endemic situation of typhoid fever in Valparaiso is :

$$
\begin{aligned}
& \delta_{1}=0.02 ; \quad \delta_{2}=0.02 ; \gamma=0.111 ; p=0.017 \\
& \lambda_{11}=0.1325 ; \quad \lambda_{21}=7.794 ; \quad \lambda_{12}=0 \quad \lambda_{22}=0
\end{aligned}
$$




$$
w_{1}=0.2 ; \quad w_{2}=0.2
$$

With this parameter values we can calculate:

$$
y_{1}^{*}=0.0040 ; y_{2}^{*}=0.0049
$$

. The values of the threshold parameter $\sigma$ can then be calculated for each intensity of the vaccination strategy of food manipulators. For example :

$$
\sigma_{0}=1.1726 ; \quad \sigma_{10}=0.806 ; \quad \sigma_{50}=0.3583 ; \quad \sigma_{100}=0.21145 \text {. }
$$

Where the subindices indicate the yearly percentage of immunization by vaccination of the corresponding population. We note that, even a very low level of immunization ( 10 percent ), yields to the disease-free state. In order to estimate the time necesary to reduce the endemic level we have simulated the behavior of the system for three vaccination intensities (see Fig 1) :

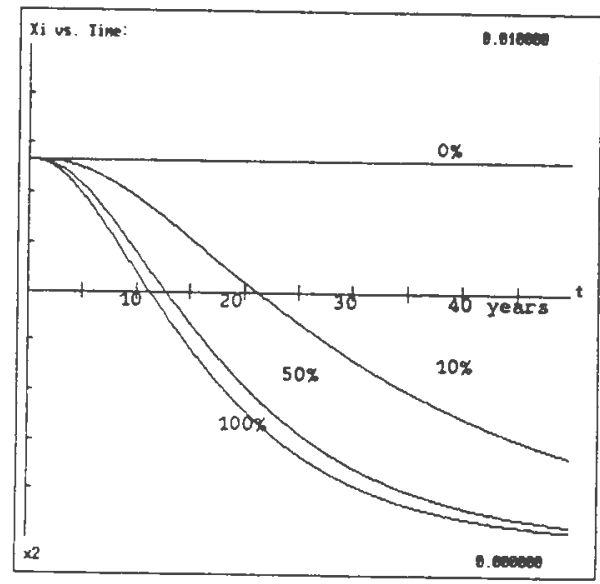

Fig 1

In order to compare the effect of a vaccination policy in the whole population see Fig 2 : 


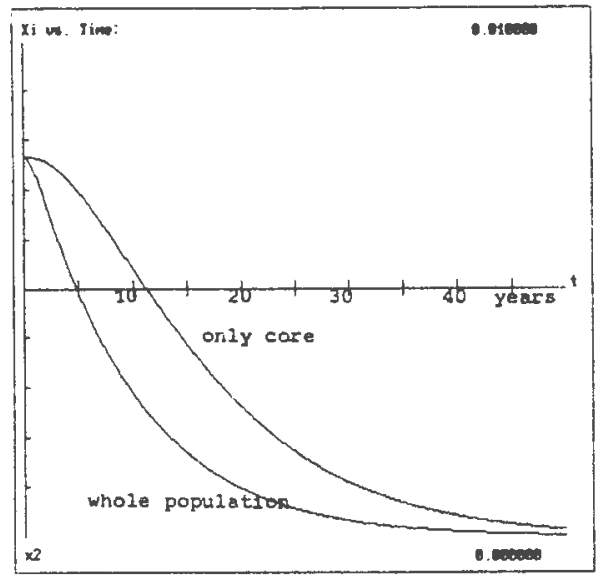

F1g 2

\section{Discussion.}

Typhoid fever is still a severe disease in our country. After a surprising increase of the endemic level from 50 reported cases per 100.000 habitants in 1960 to 120.2 in 1978 , the number of cases has decreased during the last ten years to 5652 ( 45.1 per 100.000 liabitants) in 1987 . Hence we have only returned to the old level. This endemic level is too high if we compare with other countries (for example Argentina has a rate of about 2 per 100.000 and U.S.A. has a rate of 0.2 per 100.00 ). Moreover, considering an historical undernotification of 50 percent, the real endemicity is about 100 cases per 100.000 habitants.

In the last years several control policies have been proved, in particular the Ty21a oral vaccine of René Germanier [10]. This vaccine has a 69 percent efficacy for at least four years but only if it is applied with three doses, while adding a fourth dose enhance significantly the protection [10]. Therefore is very important to estimate the possibility of focalize the control to the most important contagion agents: the food manipulators. The mor' 'dic ussed in this paper consider precisely this questiun.

The computer simulations presented liere have only an illustrative character, 'Jecause the model is still too simple and parameter estimation are not enough accurate to be practical. Nevertheless they give, in my opinion, an interesting orientation about the complex problem of controlling this disease.

\section{REFERENCES}

[1] Hethcote, H.; Yorke, J.: Gonorrhea: Transmission Dynamics and Control. Lecture Notes in Biomathematics 56.Springer Verlag, 1984 
[2] Hethcote, II: Qualitative analysis of conmunicable disease models. Math. Biose., 28, (1976), 335-356.

[3] Hethcote, H.: All inmunization model for a heterogeneous population. Theoret.Population Biol., 14 (1978), 338-349.

[4] Thieme, It.R.: Global asyınptotic stability in epidemic models. Equadiff, Lecture Noles in Math. 1017, Springer Vellag, Heildelberg, (1983), 608-615.

[5] Hethcote, H.; Thieme, H.: Stability of the Endemic Equilibrium in Epidenic Models with Subpopulations. Math.Biosc. 75:205-227 (1985).

[6] González-Guzmán, J.: An epidemiological model for direct and indirect transinission of typloid fever. Math.Biosc. 96 (1989), 33-46.

[7] González-Guzmán, J.; Naulin, R.: Analysis of a model of bovine brucellosis using singular perturbations. Pre print.

[8] Beretta, E.; Cappaso, V.: Global stability results for a multigroup SIR Epidenic Model. Proc.of the Autumn Course Reseach Seminars on Mathematical Ecology. ICTP, Trieste, 1986.

[9] Jordall, D.W.; Smith : Nonlinear Ordinary Differential Equations, Oxford Univ. Press, 1977.

[10] Levine, M.; Ferreccio, C.; Black, R.; Tacket, C.; Germanier, R.: Progress in Vaccines Against Typhoid Fever. R. of Infectious Diseases, Vol.11 MayJune 1989.

Received: September 30, 1993.

Jorge González-Guzmán and Betsabé González-Yañez

Instituto de Matemática

Universidad Católica de Valparaíso

Casilla 4059, Valparaíso, Chile. 